\title{
Spindle Cell Tumor in Oral Cavity: A Rare Case Report
}

\author{
Richa Wadhawan', Nisheeth Saawarn², Swati Saawarn³, Dharmendra Sharma ${ }^{4}$ \\ ${ }^{1}$ Reader, Department of Oral Medicine and Radiology, Institute of Dental Education and Advance Studies, Gwalior, Madhya \\ Pradesh, ${ }^{2}$ Professor and Head of the Department, Department of Oral Medicine and Radiology, Bhabha College of Dental \\ Sciences, Bhopal, MP, ${ }^{3}$ Reader, Department of Oral Pathology and Microbiology, Peoples Dental Academy, Bhopal, MP, \\ ${ }^{4}$ Tutor, Department of Oral Medicine and Radiology, Institute of Dental Education and Advance Studies, Gwalior, Madhya \\ Pradesh, India
}

Corresponding author: Dr. Richa Wadhawan, Reader, Dept of Oral Medicine and Radiology. Institute of Dental Education and Advance Studies, Gwalior, Madhya Pradesh, India.

DOI: http://dx.doi.org/10.21276/ijcmsr.2018.3.4.39

How to cite this article: Richa Wadhawan, Nisheeth Saawarn, Swati Saawarn, Dharmendra Sharma. Spindle cell tumor in oral cavity: a rare case report. International Journal of Contemporary Medicine Surgery and Radiology. 2018;3(4):D172-D175.

\section{A B S T R A C T}

Introduction: Spindle cell neoplasms of oral cavity are rare and aggressive pathological entity having epithelial, mesenchymal and odontogenic components. Being very difficult to diagnose in routine investigations these neoplasms can be diagnosed from immunohistochemistry. Management includes combination of surgery and radiotherapy.

Case report: This article aims to report a case of the spindle cell neoplasm of the oral cavity in a 50 year old female with emphasis on histopathology.

Conclusion: Appropriate diagnosis is indispensable for formulating correct treatment plan in order to minimize postoperative morbidity and mortality.

Key words: Spindle Cell, Neoplasms, Oral Cavity, Spindle Cell Carcinoma, Squamous Cell Carcinoma, Immunohistochemistry

\section{INTRODUCTION}

Spindle cell lesions affecting oral mucosa are quite diverse in nature and their diagnosis is always a challenge. While some are malignant, others are benign or simply reactive in nature. Spindle cell carcinoma (SpCC) mimicks squamous cell carcinoma (SCC) in which the spindle epithelial cell resembles a sarcoma on histological examination. Various other terminologies coined are pseudo sarcoma, carcinosarcoma, sarcomatoid squamous cell carcinoma or polypoid squamous cell carcinoma. ${ }^{1}$ Neoplasm may manifest as polypoid, pedunculated neoplasm, protruding from the mucosal surface with ulceration. Most commonly affected sites in the head and neck are oral cavity, larynx, tonsil and pharynx. Males are more commonly affected than females. It mainly occurs in fifth and sixth decade of life affecting many other sites of oral cavity. The predisposing factors for the $\mathrm{SpCC}$ are the same as that of SCC like tobacco, alcohol, poor oral hygiene and history of irradiation. Oral clinician poses a diagnostic dilemma and challenge in ruling out spindle cell neoplasm with SCC. ${ }^{2}$ No classification of this lesion has been evident in the literature till date. [Table 1]. A useful classification for cytopathologists is proposed on the predominance of spindle cells in the histopathology of the lesions of the oral cavity and it includes neural, myofibroblastic, muscle, fibroblastic, vascular, epithelial, odontogenic and miscellaneous tumors. ${ }^{3}$

Neural tumors
Neurofibroma
Neurilemmoma (schwannoma)
Palisaded encapsulated neuroma
Traumatic neuroma (amputation neuroma)
Malignant peripheral nerve sheath tumo
Myofibroblastic tumors
Myofibroma
Inflammatory myofibroblastic tumor
Low-grade myofibrosarcoma
Muscle tumors
Leiomyoma
Vascular leiomyoma
Leiomyosarcoma
Rhabdomyoma
Rhabdomyosarcoma
Fibroblastic tumors
Solitary fibrous tumor
Fibromatosis
Nodular fasciitis
Desmoplastic fibroma
Fibrosarcoma

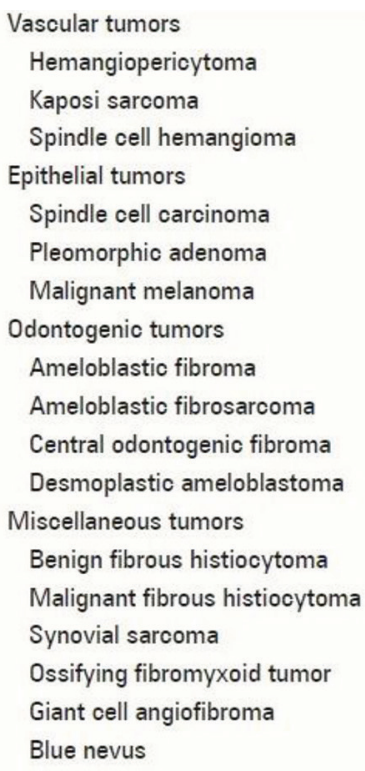

\section{CASE REPORT}

A 50-year-old female patient reported to outpatient department Oral Medicine and Radiology, Institute of Dental Education and Advance Studies, Gwalior, Madhya Pradesh with a chief complaint of painful swelling in the mandibular anterior region since two months. Medical, family history and 


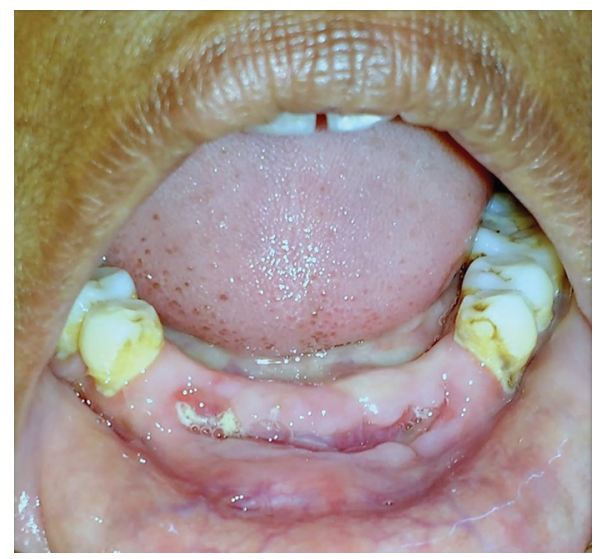

Figure-1: Photograph of sickle shaped swelling obliterating the anterior mandibular vestibule irt 313233414243

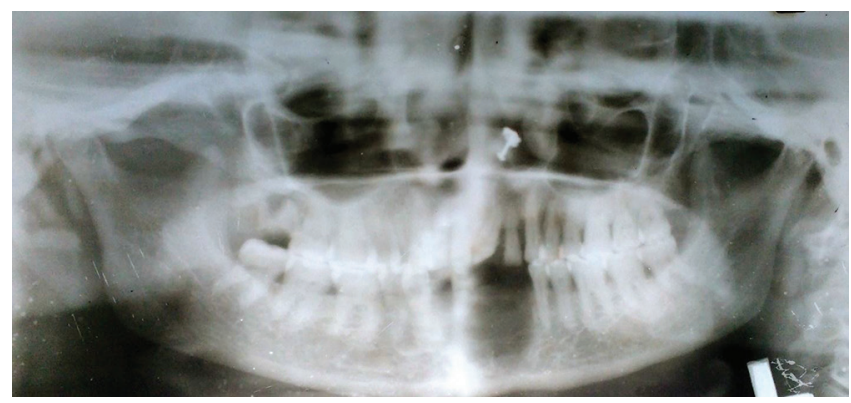

Figure-2: Panoramic radiograph revealing missing 3132 33414243 and loss of trabecular pattern in mandibular anterior region

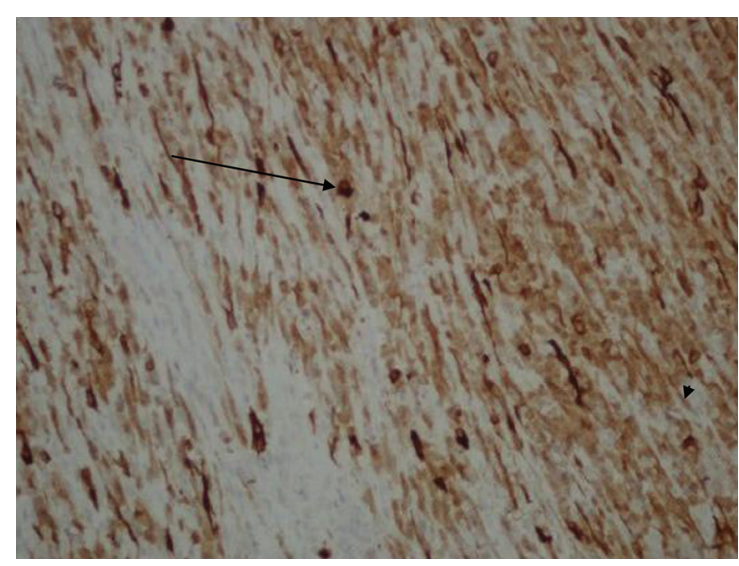

Figure-3: Photomicrograph showing diffuse and moderately intense positivity of spindle cells for pan cytokeratin

personal history were not contributory. Patient gave history of extraction of both the mandibular central, lateral incisors and canines at a private clinic one month before and soft tissue was curetted from the socket and multiple grey brown soft tissue pieces measuring $1.5 \mathrm{X} 1 \mathrm{X} 1 \mathrm{~cm}$ was submitted for histopathological evaluation. Histopathological examination of the excised specimen revealed a cellular connective tissue stroma with fascicular arrangement of elongated, spindle shaped cells embedded in connective tissue stroma exhibiting mild to moderate nuclear and cellular atypia. No necrosis evident in section examination. There was evidence of regional lymphadenopathy. Submental, Right and left submandibular lymph nodes were palpable. Submental lymphnodes were firm on palpation. Extra oral examination revealed diffuse midline swelling present in submental region. Intraoral examination revealed unhealed socket irt 3132 33414243 and sickle shape soft tissue swelling extending from lower left canine to right canine with overlying surface same as that of normal skin color in mandibular labial vestibule in same region of size $3.5 \mathrm{~cm} \times 3.5 \mathrm{~cm}$. (Fig 1) Other oral features include generalized gingival recession and root stump irt 18. So Panoramic radiograph was advised. Radiographic investigations revealed generalized bone loss and loss of trabecular pattern in mandibular anterior region. (Fig 2). Based on above mentioned findings it was diagnosed as spindle cell neoplasm. The differential diagnoses considered were spindle cell carcinoma, spindle cell melanoma and mesenchymal neoplasms like fibro sarcoma. Patient was referred to department of oral and maxillofacial surgery for excisional biopsy. Excisional biopsy of mass was performed under local anaesthesia. To confirm the diagnosis immunohistochemical analysis was done. The tumour cells were positive for the markers AE1/AE3, focally positive for EMA (Fig 3) while negative for S-100, myogenin, desmin and p63. Based on these findings, a final diagnosis of spindle cell carcinoma was made. The patient was lost to follow-up and hence no further treatment was possible.

\section{DISCUSSION}

Spindle cells are mesenchymal in origin and are spindle cell tumors are usually described as a spindle cell variant of another common malignancy, such as squamous cell carcinoma, melanoma, or sarcomas. Spindle cell tumors are rare in the maxillofacial region and usually harbor a wide range differential diagnosis including lesions which are both benign and malignant in nature. ${ }^{4} \mathrm{SpCC}$ of the oral cavity can be diagnosed in younger age group. Our case report is of young adolescent. ${ }^{5}$ It has a site predilection for the lower lip, tongue and alveolar ridge or gingiva. In our case, the growth was seen in the vestibule of the anterior mandible. ${ }^{6}$ Lesion can often be exophytic, penduculated, polypoid, but sessile, nodular or endophytic configuration has also been reported. It usually shows an extensive surface ulceration with friable, fibrinoid necrosis of variable thickness or shaggy exudates. As discussed earlier and similar to SCC tobacco use, alcohol abuse, history of irradiation and chronic trauma seems to play a role in its etiology. ${ }^{7}$ Viswanathan et al., in an extensive clinicopathologic review of 103 cases of sarcomatoid carcinoma of the head and neck demonstrated that tobacco chewing (63.8\%) was more frequently observed than smoking $(21.3 \%)$ in these patients and none of them had past history of radiation exposure. ${ }^{8} \mathrm{SpCC}$ can demonstrate varied histopathological appearance like epithelial cells or may appear as atypical mesenchymal cells within different areas of the same tumor tissue making it extremely difficult to assess whether it is carcinoma and/ or sarcoma. The tumor generally consists of fascicles of anaplastic spindle cells with considerable number of mitotic figures. ${ }^{9}$ Differential diagnosis includes a number of benign and malignant tumors, such as fibromatosis, nodular fasciitis, reactive epithelial proliferations, SCC, fibro sarcoma, malignant fibrous histocytoma, leiomyosarcoma, 
rhabdomyosarcoma, malignant peripheral nerve sheath tumor, mesenchymal chondrosarma and malignant melanoma. ${ }^{10}$ Management of SpCC has been controversial. Wide excision including radical dissection along with metastasis is optimal treatment. Radiotherapy can be used as an adjunct in cases where surgery alone is not sufficient. Vital role of radiotherapy has been evident in cases with positive surgical margin positive or extensive nodal disease. Prognosis is poor. Mortality rate within one year of life has been found in $30 \%$, which is similar to the high grade SCC. As per study conducted by Ellis and Corio presence of metastasis signals a poor prognosis. ${ }^{11}$ Recurrence rate after surgical excision has been found around $75 \%$ and metastasis rate around $35 \% .{ }^{12}$ Spindle cell neoplasms of the oral cavity form a diverse group and it is very difficult to diagnose these neoplasms from routine haemotoxylin and eosin sections of histopathology. Histopathologic findings showed a connective tissue stroma infiltrated with numerous spindle shaped tumor, many of them round to oval in shape, with eosinophilic and vacuolated cytoplasm. They showed prominent anisocytosis, anisonucleosis with more than one nucleoli, hyperchromatism, and frequent mitotic figures. Alcian blue staining indicated myxomatous areas. These features suggested a malignant neoplasm of spindle cell category. Immuno histochemical investigations provide a definite diagnosis and helps in ruling out individual neoplasms. ${ }^{13}$ Positive reaction to markers such as keratin, vimentin, and S-100 protein are very crucial in differentiating $\mathrm{SpCC}$ from true spindle cell sarcoma, melanoma and malignant myoepithelioma. The point to note is that SpCC should not be ruled out by a positive reaction for vimentin in sarcomatoid tumour cells. No sign of staining for keratin in the sarcomatoid tumour cells not necessarily excludes SpCC, because some lesions of SpCC show immunoreactivity of keratin in their sarcomatoid components only with some anti-keratin antibodies. Differential diagnosis of SpCC requires various kinds of anti-keratin antibodies. IHC analysis of our cases shows co-expression of cytokeratin $(\mathrm{CK})$ and vimentin to various degrees. Microscopic features may exhibit only the monophasic sarcomatoid component or biphasic features. and The chief feature of spindle cell component includes elongated cells, hyper chromatic nuclei with numerous mitotic figures and the squamous component found mostlyis non keratinizing (poorly differentiated). The chief differential diagnoses of $\mathrm{SpCC}$ are true superficial sarcomas and benign but atypical reactive angiofibroblastic or myofibroblastic proliferations involving the mucosa. ${ }^{14} \mathrm{Being}$ rare sarcomas and reactive pseudosarcomatous proliferations arising from the sub mucosa renders the assumption that a polypoid mucosal malignant spindle cell proliferation is a $\mathrm{SpCC}$ is reasonable. Simultaneous expression of cytokeratin (CK) and vimentin in IHC analysis using polymer HRP detection system and $\mathrm{DAB}$ chromogen show concurrent presence of malignant epithelial and sarcomatoid spindle cell components. Negative immunoreactivity to S-100 protein has been demonstrated in all cases of $\mathrm{SpCC}$. A major tool in classification of epithelial neoplasm is waterinsoluble proteins called as cytokeratins and Vimentin in a variety of mesenchymal cells. ${ }^{15}$

\section{CONCLUSION}

Spindle cell lesions of either carcinomatous or sarcomatous origin are diagnostically challenging group of lesions. Appropriate diagnosis is indispensable for formulating correct treatment plan in order to minimize post-operative morbidity and mortality. Further, it is essential that in the absence of advanced diagnostic aids, a microscopically diagnosed spindle cell lesion should be considered to be of epithelial origin and treated likewise unless proved otherwise.

\section{REFERENCES}

1. Anderson CE, Al-Nafussi A. Spindle cell lesions of the head and neck: an overview and diagnostic approach. Diagn Histopathol. 2009; 15(5):264-272.

2. Thompson LDR. Squamous cell carcinoma variants of the head and neck. Curr Diagn Pathol. 2003; 9:384-396.

3. Thompson LD, Wieneke JA, Miettinen M, Heffner DK. Spindle cell (sarcomatoid) carcinomas of the larynx: a clinicopathologic study of 187 cases. Am J Surg Pathol. 2002; 26(3):153-170.

4. Katase N,Tamamura R, Gunduz M,MurakamiJ,Asaumi J, Tsukamoto G, Sasaki A, Nagatsuka H. A spindle cell carcinoma presenting with osseous metaplasia in the gingiva: a case report with immunohistochemical analysis. Head Face Med. 2008; 4:28.

5. Munakata R, Cheng J, Nakajima T, Saku T. Spindle cell carcinoma of the gingiva: report of an autopsy case. J Oral Pathol Med. 1998; 27(4):180-184.

6. Chen YK, Lin CC, Chen CH, Yan YH, Lin LM. Spindle cell carcinoma of the tongue: case report and immunohistochemistry study. Oral Med Pathol. 1998; 3(4):51-54.

7. Batsakis JG, Suarez P. Sarcomatoid carcinomas of upper aerodigestive tracts. Adv Anat Pathol. 2000; 7:282-293.

8. Seethalakshmi V, Khaliqur R, Suryawanshi P, Johari S, Asawari P, Pankaj C, Anil D Cruz, Jaiprakash A, and S. V. Kane. Sarcomatoid (Spindle Cell) Carcinoma of the Head and Neck Mucosal Region: A Clinicopathologic Review of 103 Cases from a Tertiary Referral Cancer Centre. Head Neck Pathol. 2010; 4(4): 265-275.

9. Su HH, Chu ST, Hou YY, Chang KP, Chen CJ. Spindle cell carcinoma of the oral cavity and oropharynx: factors affecting outcome. J Chin Med Assoc. 2006; 69(6):478483.

10. Takata T, Ito H, Ogawa I, Miyauchi M, Ijuhin N, Nikai $\mathrm{H}$. Spindle cell squamous carcinoma of the oral region. An immunohistochemical and ultrastructural study on the histogenesis and differential diagnosis with a clinicopathological analysis of six cases. Virchows Arch. 1991; 419(2):177-182.

11. Ellis GL, Langloss JM, Heffner DK, Hyams VJ. Spindle-cell carcinoma in the upper aerodigestive tract. An immunohistochemical analysis of 21 cases. Am J Surg Pathol. 1987; 11(5):335-342.

12. Hellquist H, Olofsson J. Spindle cell carcinoma of the larynx. APMIS. 1989; 97(1):1103-1113.

13. Hyams VJ. Spindle cell carcinoma of the larynx. Can J Otolaryngol. 1975; 4(2):307-313.

14. Lambert PR, Ward PH, Berci G. Pseudosarcoma of the larynx: a comprehensive analysis. Arch Otolaryngol. 
1980; 106(5):700-708.

15. Olsen KD, Lewis JE, Suman VJ. Spindle cell carcinoma of the larynx and hypopharynx. Otolaryngol Head Neck Surg. 1997; 116(6):47-52.

16. Sherwin RP, Strong MS, Vaughn CW., Jr Polypoid and junctional squamous cell carcinoma of the tongue and larynx with spindle cell carcinoma ('pseudosarcoma') Cancer. 1963; 16(1):51-60.

Source of Support: Nil; Conflict of Interest: None

Submitted: 18-10-2018; Accepted: 02-12-2018; Published online: 11-01-2019 